\title{
Analytical Modeling of RRM-ATM Switch for Linear Increment and Exponential Decay of Source Data Rate
}

\author{
Mohsen Hosamo ${ }^{1, *}$, S. P. Singh ${ }^{2}$, Anand Mohan ${ }^{2}$ \\ ${ }^{1}$ Oslo, Norway \\ ${ }^{2}$ Department of Electronics Engineering, Indian Institute of Technology (BHU), Varanasi, India \\ *Corresponding author
}

\begin{abstract}
This paper describes analytical modeling of relative rate marking (RRM) switch for available bit rate (ABR) ATM service considering linear increment and exponential decay of data rate(s) of the source(s) to achieve faster congestion control of the network through resource management of the RRM switch. Theoretical performance of the switch has been evaluated in respect of link utilization factor and cell loss probability. It is shown that the switch achieves faster control over source(s) along with improved link utilization factor. This is particularly attractive for congestion control in ABR-ATM networks. The developed switch model can find potential application in the design of RRM switch specific to a network environment.
\end{abstract}

\section{Keywords: ATM Switch, RRM Switch Architecture, Congestion Control, RRM Switch Performance}

Cite This Article: Mohsen Hosamo, S. P. Singh, and Anand Mohan, "Analytical Modeling of RRM-ATM Switch for Linear Increment and Exponential Decay of Source Data Rate." Journal of Computer Networks, vol. 4, no. 1 (2017): 56-64. doi: 10.12691/jcn-4-1-6.

\section{Introduction}

Architecture and performance parameters of asynchronous transfer mode (ATM) switches [1,2,3] greatly influence the quality of service (QoS) of ATM networks. This has been the major driving force for continued interest in the development of variety of ATM switches having their inherent merits for specific applications. Essentially relative rate marking (RRM) switches achieve speed superiority over explicit forward congestion indication (EFCI) by making use of congestion indication (CI) and no increase (NI) bits of resource management (RM) cells for congestion control [4]. Further, RRM switches are obvious choice and thus being extensively used in ATM networks as they can operate with finer resolution and provide a good trade off between the congestion control time and hardware complexity [6,9]. In spite of attractive features, RRM switches are not yet fully explored nor optimized under various operational network parameters. Mars et al. used an RRM switch to investigate the performance of transmission control protocol (TCP) connection [7], Lapsley and Rumsewicz [8] discussed advantages and disadvantages of using NI bit in the feedback loop to control the source data rate. Mischa Schwartz [10] discussed another feedback method for congestion control in broadband networks and Plotkin and Sydir proposed an implementation of RRM switch [11].

This paper bridges the gap of knowledge about performance of RRM switches by developing its mathematical model which provides theoretical foundation for RRM switch analysis. Theoretical analysis of maximum and minimum queue length for determination of Available Bit Rate (ABR) and resource management delays is presented followed by computation of queue length, time delay parameters, link utilization $(\rho)$ and cell loss probability ( $P_{\text {loss }}$ ) for a given QoS.

\section{Switch Modeling}

Figure 1 shows the basic RRM switch architecture, which contains a multiplexer (MUX) and de-multiplexer (DMUX) at the input and output respectively and a shared memory used as queue $(Q)$ to store data cells of sources. The $Q$ is an important shared resource of RRM switch whose status is monitored for source data rate control using feedback loop. Therefore the techniques used for $Q$ implementation and its allocation have significant impact on the overall ATM performance which warrants development of an appropriate theoretical model for analysis of the switch and its effect on the ATM network performance.

\subsection{Analogy with Fluid Flow}

Considering the shared memory of Figure 1 as a bucket having an inlet-outlet in which the cells are poured as fluid that comes out from outlet. Assuming there are "N" sources each having same allowed cell rate ( $A C R$ ), the total rate of incoming cells " $x\left(t-\tau_{f}\right)$ " at time "t" can be given as “ $N \times A C R\left(t-\tau_{f}\right)$ " where $\tau_{f}$ is delay time of forward resource management cells (FRMC). Considering that output (or service) rate is $\mu$ then fluid flow approximation of the $Q$ can be represented as in Figure 2 which shows the total incoming cell rate 
$x\left(t-\tau_{f}\right)$, outgoing cell rate $\mu=L C R$ (available link cell rate), net rate of increase/decrease in $Q$ will be "[ $\left.N \times A C R\left(t-\tau_{f}\right)-L C R\right]$ ]. Therefore the rate of change of queue length “ $Q(t)$ ” at time $t$ can be written as $\frac{d Q(t)}{d t}=N \times A C R\left(t-\tau_{f}\right)-L C R$ and hence the value of $Q(t)$ at any instant $t$ can be expressed as

$$
Q(t)=Q\left(\tau_{f}\right)+\int_{\tau_{f}}^{t}\left[N \times A C R\left(t-\tau_{f}\right)-L C R\right] d t .
$$

\subsection{Analysis of $Q(t)$ and $A C R$}

The network model of the RRM switch having shared memory $Q$ is shown in Figure 3 along with definition of the relevant parameters given in Table 1 . Figure 4 gives the simplified data flow view of ATM network indicating forward and backward resource management cells (FRMC and BRMC) and actual data cells. Under operating conditions, any change in the rate of data cells affects the rate of $B R M C$ and the bounds of the source rate would be $M C R \leq A C R(t) \leq P C R$, where $M C R$ and
PCR are the minimum and peak cell rates respectively. The steps in which the cell rate of sources can be increased is $1 / 2^{n}$ [12], where $n$ is a positive integer and this step size is called rate increase factor ( $R I F$ ). Therefore increase in cell rate at any instant $t$ would be $(R I F \times P C R)$ and for FRMC it shall be (PCR.RIF)/ Nrm, where Nrm is number of data cells between two FRMCs. Therefore the elemental increase in allowed cell rate $A C R(t)$ for one source can be given as:

$$
\frac{d A C R(t)}{d t}=(P C R . \times R I F / N r m)(I C R) .
$$

Integrating equation (2) and using boundary condition $A C R(t)=A C R(0)$ at $t=0$ gives

$$
A C R(t)=\{A C R(0)+(P C R \times R I F / N r m)(I C R) \times t\} .
$$

Therefore the value of $A C R(t)$ at any instant can be found by considering its bounds as

$$
\begin{aligned}
& A C R(t) \\
& =\operatorname{Min}[P C R,\{A C R(0)+(P C R \times R I F)(I C R) \times t\}] .
\end{aligned}
$$

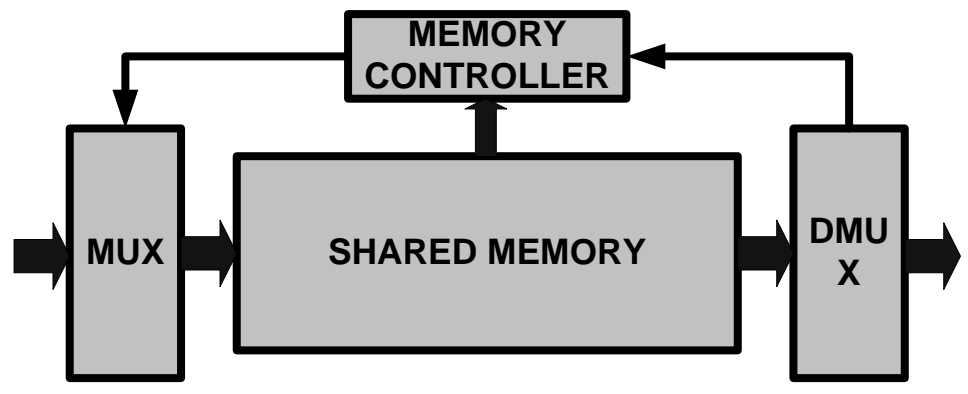

Figure 1. Shared Memory Architecture

$x\left(t-\tau_{f}\right)=N \times \operatorname{ACR}\left(t-\tau_{f}\right)$

Input_Rate

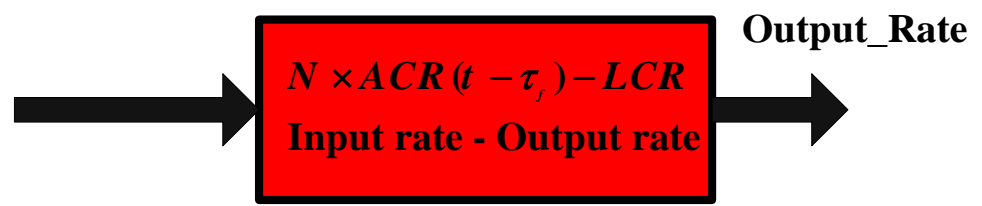

Figure 2. Fluid Flow Model for Shared Memory Queue

Table 1. The definition of the relevant parameters

\begin{tabular}{|l|l|}
\hline ICR & Initial Cell Rate \\
\hline$A C R_{\max }$ & Maximum Value of ACR. \\
\hline$A C R_{\min }$ & Minimum Value of ACR. \\
\hline$Q_{\max }$ & Total buffer size \\
\hline$Q_{H}$ & Higher value of Queue length threshold for congestion \\
\hline$Q_{L}$ & Indication about lower value of queue length threshold \\
\hline$\tau_{f}$ & Time Delay from the queue to the source \\
\hline$\tau_{b}$ & Time Delay from the Switch to the Source \\
\hline$\tau_{A C R_{\max }}$ & Time Required for Attaining cell rate of $A C R_{\text {max }}$ from ICR. \\
\hline$\tau_{A C R_{\min }}$ & Time Required for Attaining cell rate of $A C R_{\min }$ from ICR \\
\hline
\end{tabular}




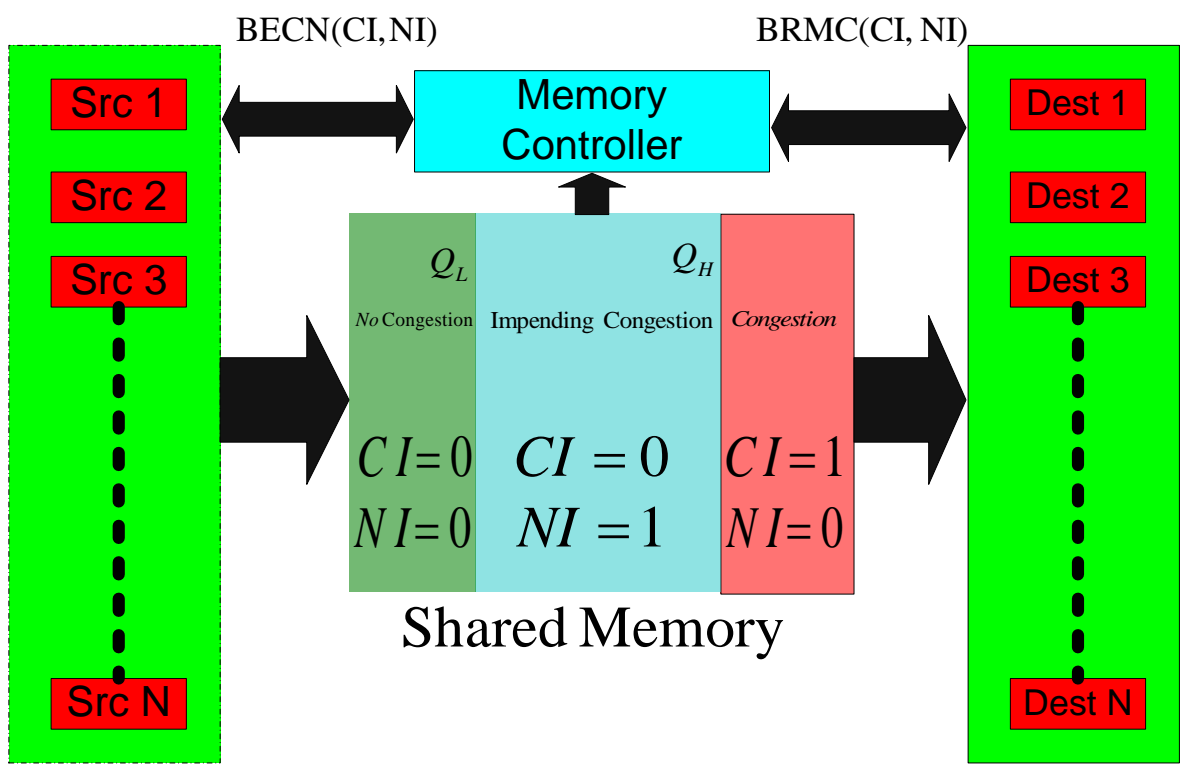

Figure 3. Network Model

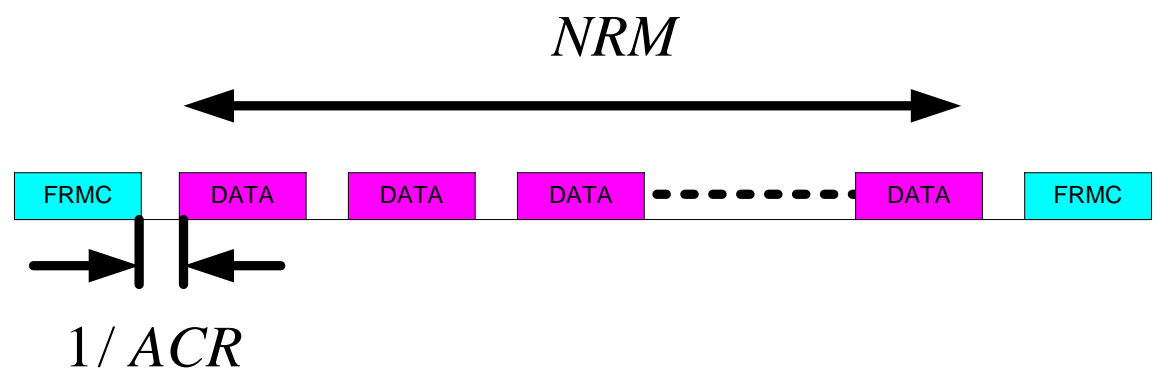

Figure 4. Data and FRM Cells flow

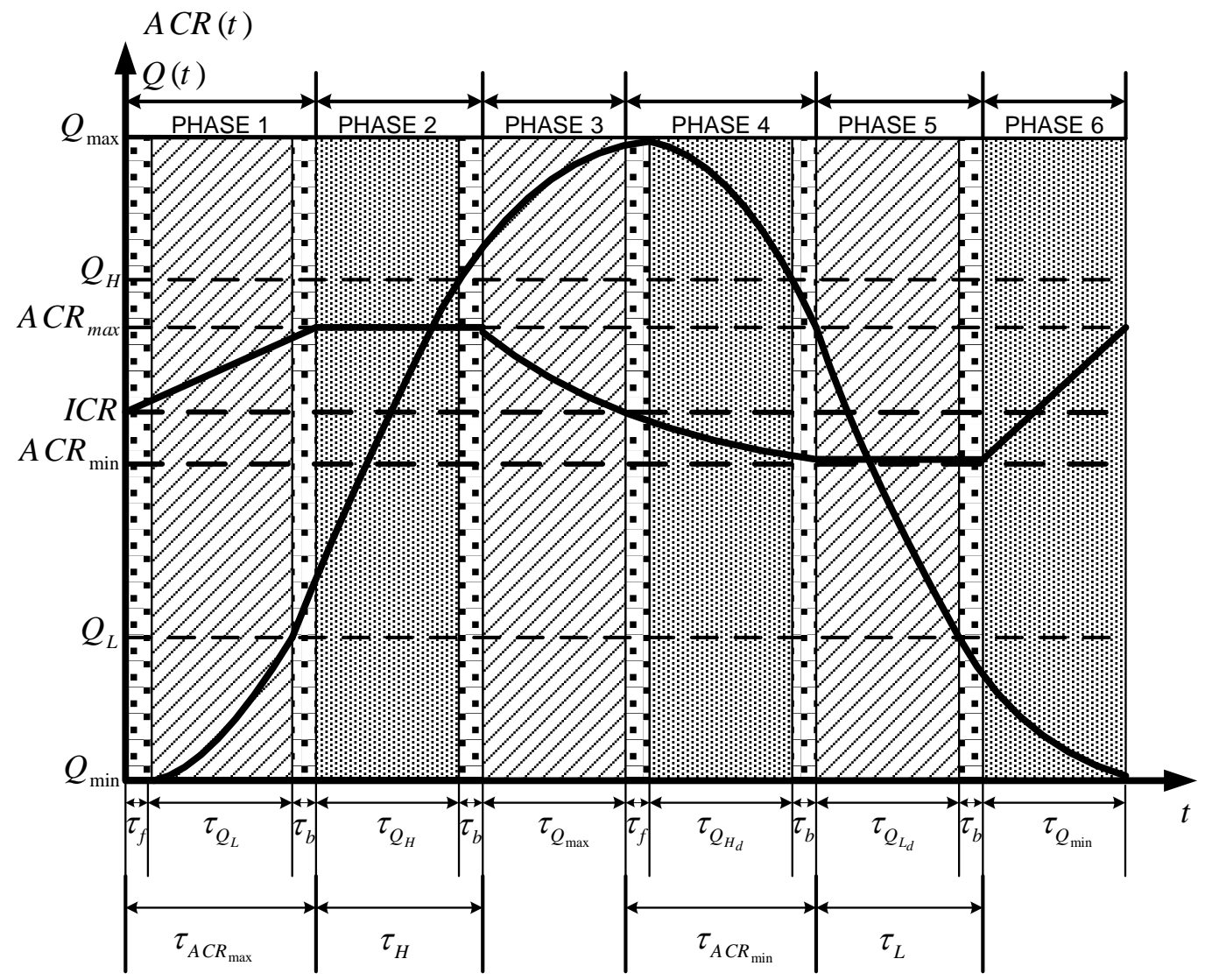

Figure 5. Variation in $Q(t)$ and $A C R(t)$ as function of time 
The variations of $Q(t)$ and $A C R(t)$ computed using equations (1) and (4) respectively are plotted as function of time in Figure 5 from which it is seen that $Q(t)$ has six operational phases between its limiting values $Q_{\min }$ and $Q_{\max }$ as discussed below.

Phase (i): Initially at $t=0$, the source starts sending data and $Q(t)$ starts building up after delay $\tau_{f}$ until the $Q$ is filled up to $Q_{L}$ and when $Q(t)=Q_{L}, B R M C$ is sent with $\mathrm{CI}=0$ and $\mathrm{NI}=1$ to fix the data rate of the source to $A C R_{\text {max }}$ after a time delay of $\tau_{b}$. Assuming that $A C R(t)$ is increasing linearly from $I C R$ to its upper limit $P C R$ (peak cell rate) $=A C R_{\max }$ in the worst case then the value of $A C R_{\max }$ can be written as

$$
\begin{aligned}
& A C R_{\max }=P C R \\
& =I C R+\frac{R I F \times P C R \times I C R}{N r m} \tau A C R_{\max } \\
& \text { and thus } \tau_{A C R_{\max }}=\frac{N r m(P C R-I C R)}{(R I F)(P C R)(I C R)}
\end{aligned}
$$

and for special case $I C R=\frac{L C R}{N}$ then

$$
\tau_{A C R_{\max }}=\frac{N r m(N \times P C R-L C R)}{(R I F)(P C R)(L C R)} .
$$

For $A C R_{\max }<P C R, Q_{L}$ can be expressed as

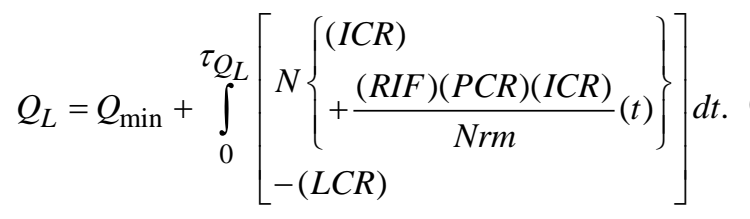

Therefore using equation (7), $\tau_{Q_{L}}$ can be expressed as

$$
\tau_{Q_{L}}=\frac{\left[\begin{array}{l}
-(N . I C R-L C R) \\
\pm \sqrt{(N \cdot I C R-L C R)^{2}}+4\left(\frac{N \cdot R I F \cdot P C R \cdot I C R}{2 \cdot N r m}\right)\left(Q_{\min }-Q_{L}\right)
\end{array}\right]}{2 \cdot \frac{N \cdot R I F \cdot P C R \cdot I C R}{2 \cdot N r m}}
$$

and for

$$
I C R=\frac{L C R}{N}, \tau_{Q_{L}}=\sqrt{\frac{2 N r m\left(Q_{L}-Q_{\min }\right)}{(R I F)(P C R)(L C R)}} .
$$

Therefore the maximum time delay for change in cell rate from $I C R$ to $A C R_{\max }$ can be obtained as

$$
\tau_{A C R_{\max }}=\tau_{Q_{L}}+\tau_{b}+\tau_{f} .
$$

Phase (ii): When $Q(t)$ equals $Q_{H}, A C R(t)$ starts decreasing after lapse of time $\tau_{b}$, which is needed by the $B R M C$ (with $\mathrm{CI}=1, \mathrm{NI}=0$ ) to arrive at the source. Therefore $Q_{H}$ can be written as

$$
\begin{gathered}
Q_{H} \\
=Q_{\min }+\int_{0}^{\tau A C R_{\max }}\left[N\left\{\begin{array}{l}
(I C R) \\
+\frac{(R I F)(P C R)(I C R)}{N r m}(t)
\end{array}\right\}\right] d t(11) \\
\left.+\int_{0}^{\tau_{Q_{H}}}[N(A C R \text { max }))-(L C R)\right] d t \\
Q_{H}=Q_{\min }+\frac{N(R I F)(P C R)(I C R)}{2 N r m}\left(\tau_{A C R_{\max }}^{2}\right) \\
+N\left(A C R_{\max } \times \tau_{Q_{H}}\right)-(L C R)\left(\tau_{Q_{H}}\right) .
\end{gathered}
$$

Using equation (5) and substituting the value of $\frac{N(R I F)(P C R)(I C R)}{2 N r m} \tau_{A C R_{\max }}^{2}$ into equation (12), we have

$$
\begin{aligned}
Q_{H}= & Q_{\min }+\frac{N \times A C R_{\max }}{2} \tau_{A C R_{\max }}-\frac{N(I C R)}{2 . N} \tau_{A C R_{\max }} \\
& +N\left(A C R_{\max } \times \tau_{Q_{H}}\right)-(L C R)\left(\tau_{Q_{H}}\right) .
\end{aligned}
$$

For $I C R=\frac{L C R}{N}$,

$$
\begin{aligned}
& Q_{H} \\
& =Q_{\min }+\left(\frac{\tau_{A C R_{\max }}}{2}+\tau_{Q_{H}}\right)\left(N \times A C R_{\max }-L C R\right)
\end{aligned}
$$

Therefore the time required to change $Q(t)$ between $Q_{H}$ and $Q_{\max }$ i.e. $\tau_{Q_{H}}$ can be found using equation (15) as

$$
\tau_{Q_{H}}=\frac{Q_{H}-Q_{\min }}{N \times A C R_{\max }-L C R}-\frac{\tau A C R_{\max }}{2} .
$$

The total time delay $\tau_{H}$ during which $A C R(t)=$ $A C R_{\text {max }}=$ constant and after which the source starts reducing $A C R(t)$ can be expressed as

$$
\tau_{H}=\tau_{Q_{H}}+\tau_{b}
$$

Phase (iii): When $A C R(t)$ decreases from $A C R_{\max }$ to $I C R$, the $Q(t)$ continues to increase until it reaches $Q_{\max }$. Therefore $Q_{\max }$ can be expressed as

$$
\begin{aligned}
& Q_{\max }= \\
& Q_{\min }+\int_{0}^{\tau A C R_{\max }}\left[N\left\{\begin{array}{l}
(I C R) \\
-(L C R) \\
-(R I F)(P C R)(I C R) \\
N r m
\end{array}\right\}\right] d t \\
& +\int_{0}^{\tau_{H}}[(N \times A C R \max )-(L C R)] d t \\
& +\int_{0}^{\tau_{Q_{\max }}}\left[\left(N \times A C R_{\max }\right) e^{-\frac{(R D F)(I C R)}{N r m} t}-(L C R)\right] d t
\end{aligned}
$$


or

$$
Q_{\max }=Q_{1}+\int_{0}^{\tau_{Q_{\max }}}\left[\begin{array}{l}
\left(N \times A C R_{\max }\right) e^{\frac{-(R D F)(I C R)}{N r m} t} \\
-(L C R)
\end{array}\right] d t(18)
$$

where

$$
\begin{aligned}
& Q_{1} \\
& =Q_{\min }+\int_{0}^{\tau A C R_{\max }}\left[N\left\{\begin{array}{l}
(I C R) \\
-(L C R)
\end{array}\right]\right. \\
& +\int_{0}^{\tau_{H}}[(N \times A C R \max )-(L C R)] d t \\
& Q_{\max }=Q_{1}+\frac{N \times N r m}{R D F}\left[e^{\frac{(R D F)(I C R)}{N r m} \tau^{Q_{\max }}-1}\right] d t \\
& -(L C R) \tau_{Q_{\max }} .
\end{aligned}
$$

Considering the $A C R(t)$ curve of Figure 5, we have $I C R=A C R_{\max }\left\{e^{\frac{-(R D F)(I C R)}{N r m} Q_{\max }}\right\}$.

Therefore

$$
\tau_{Q_{\max }}=-\frac{N r m}{R D F . I C R} \ln \left(\frac{I C R}{A C R_{\max }}\right)
$$

and

$$
\begin{aligned}
& Q_{\max } \\
& =Q_{\min }+\int_{0}^{\tau A C R_{\max }}\left[N\left(I C R+\frac{R I F \cdot P C R \cdot I C R}{N r m} t\right)\right] d t \\
& +N . A C R_{\max } \cdot \tau_{H}-L C R \cdot \tau_{H} \\
& +\frac{N . N r m}{R D F}\left[\ln \left(\frac{I C R}{A C R_{\max }}\right)+\frac{A C R_{\max }}{I C R}-1\right] .
\end{aligned}
$$

As the rate between two FRM cells cannot be decreased by more than rate decrease factor ( $R D F$ ) [12], referring Figure 4 the decrease in FRM cell rate would be $[A C R(t) \times R D F]$ and corresponding rate decrease for Nrm data cells would be $[\{A C R(t) \times R D F\} / N r m]$. Therefore rate of decrease in data cell rate $A C R(t)$ for one source can be expressed as

$$
-\frac{d}{d t}\{A C R(t)\}=[\{A C R(t) \times R D F\} / N r m](I C R) .
$$

Solving equation (22) we get

$$
A C R(t)=\{A C R(0)\}\left\{e^{-\frac{(R D F)(I C R) t}{N r m}}\right\} .
$$

Thus the appropriate value of $A C R(t)$ can be determined as

$$
A C R(t)=\operatorname{Max}\left[\operatorname{MCR},\left\{A C R(0) \times e^{-\frac{R D F . I C R}{N r m}}\right\}\right] .
$$

There are another three phases for the $Q$ variation when it decreases from $Q_{\max }$ to $Q_{\min }$

Phase (iv): When $A C R(t)$ decreases exponentially from ICR to $A C R_{\min }, Q(t)$ starts decreasing after $\tau_{f}$ time delay when FRMC (with $\mathrm{CI}=1, \mathrm{NI}=0$ ) arrives at the switch. Assuming $A C R_{\min }=M C R, \tau_{A C R_{\min }}$ can be written as

$$
\tau_{A C R_{\min }}=\frac{-N r m}{(R D F)(I C R)} \ln \left(\frac{M C R}{I C R}\right) .
$$

For $A C R_{\min }>M C R$ the value of $\tau_{A C R_{\min }}$ is found from "Q(t)" curve of Figure 5 as

$$
\begin{aligned}
Q_{H}= & Q_{\max }+\frac{N \times N r m}{R D F}\left[e^{-\frac{(R D F)(I C R)}{N r m} \tau_{H_{d}}}-1\right] \\
& +(L C R) \tau_{Q_{H_{d}}}
\end{aligned}
$$

and time needed to reduce the cell rate to $A C R_{\min }$ is

$$
\tau A C R_{\min }=\tau_{Q_{H_{d}}}+\tau f+\tau_{b} .
$$

Phase (v): When $Q(t)$ reduces to $Q_{L}, A C R(t)$ starts increasing after delay $\tau_{b}$ when $B R M C$ (with $\mathrm{CI}=0, \mathrm{NI}=1$ ) arrives at the source. Therefore the final expression for $Q_{L}$ as shown in Figure 5 can be written as

$$
\begin{aligned}
Q_{L}= & Q_{\max }+\frac{N \times N r m}{R D F}\left[e^{-\frac{(R D F)(I C R)}{N r m} \tau_{A C R_{\min }}}-1\right] \\
& -N \times A C R_{\min } \times \tau_{Q_{L_{d}}}+L C R \times \tau_{Q_{L_{d}}} .
\end{aligned}
$$

Referring $A C R(t)$ curve of Figure 5, we have

$$
\begin{aligned}
Q_{L}= & Q_{\max }+\frac{N \times N r m}{R D F}\left[\frac{A C R_{\min }}{I C R}-1\right] \\
& -N \times A C R_{\min } \times \tau_{Q_{L_{d}}}+L C R \times \tau_{Q_{L_{d}}} .
\end{aligned}
$$

Therefore

$\tau_{Q_{L_{d}}}=\frac{\left(Q_{\max }-Q_{L}\right)+\frac{N \times N r m}{R D F}\left[\frac{N \times A C R \min }{L C R}-1\right]}{N \times A C R \text { min }-L C R}$.

The time $\tau_{L}$ during which $A C R(t)$ remains constant at $A C R_{\text {min }}$ can be found as

$$
\tau_{L}=\tau_{Q_{L_{d}}}+\tau_{b}
$$


Phase (vi): Finally when $Q(t)$ reaches $Q_{\min }$, the $A C R(t)$ increases from $A C R_{\text {min }}$ to $I C R$

Therefore

$$
\begin{aligned}
& Q_{\min } \\
& =Q_{\max }-\left\{\begin{array}{l}
\int_{0}^{\tau A C R_{\min }}\left[N . I C R . e^{-\frac{R D F . I C R}{N r m} t}-L C R\right] d t \\
+\int_{0}^{\tau_{L}}\left[N . A C R_{\min }-L C R\right] d t
\end{array}\right\}
\end{aligned}
$$

and since $A C R_{\text {min }}$ can be expressed as

$$
I C R=A C R_{\min }+\frac{R I F \cdot I C R \cdot P C R}{N r m} \tau_{Q_{\min }}
$$

thus

$$
\tau_{Q_{\min }}=-\frac{N r m\left(A C R_{\min }-I C R\right)}{\text { RIF.PCR.ICR }} .
$$

\section{Analysis of Network Parameters}

This section describes the analysis of "link utilization factor" and "cell loss probability" which are the important network parameters. These parameters have been determined using the proposed model. The link utilization factor can be computed from the knowledge of the status of $Q(t)$ at any instant. For $0<Q(t)<Q_{\max }$ the shared $Q$ buffer is fully utilized but the buffer underflow occurs when $Q(t) \leq 0$, therefore the number of waste cells

$$
N_{\text {waste }}=\tau_{Q(t) \leq 0} \cdot A C R .
$$

Therefore the waste bandwidth i.e. number of cells wasted during one cycle of queue building and depletion over the time period $(T)$ can be expressed as

$$
B W_{\text {waste }}=N_{\text {waste }} / T \text {. }
$$

The cycle time $T$ of $Q(t)$ can be expressed as

$$
T=\tau_{Q_{\max }}+\tau_{A C R_{\min }}+\tau_{L}+\tau_{A C R_{\max }}+\tau_{H}
$$

and link utilization factor " $\rho$ " can be written as

$$
\rho=1-\left(B W_{\text {waste }} / L C R\right) .
$$

From equation (37) it is clear that link utilization factor $" \rho "$ varies in the range " 0 ” to " 1 ”when the waste bandwidth " $B W_{\text {waste }}$ " changes due to change in network traffic condition from " 0 " to $L C R$. However, the cell loss occurs when $Q(t)>Q_{\max }$ that causes overflow of $Q$ buffer resulting in loss of cells. Therefore the number of cells lost “ $N_{\text {loss }}$ " in one cycle time $T$ of $Q(t)$ can be expressed as

$$
N_{\text {loss }}=\tau_{Q(t)>Q \max } \cdot A C R .
$$

Therefore the cell loss probability “ $P_{\text {loss }}$ ” can be computed from

$$
P_{\text {loss }}=\frac{N_{\text {loss }}}{\int_{0}^{T} A C R(t) d t}=\frac{N_{\text {loss }}}{T \cdot L C R-\left(N_{\text {waste }}+N_{\text {loss }}\right)} .
$$

\subsection{Analytical Results}

The values of $\tau_{A C R_{\max }}$ and $\tau_{H}$ have been computed using equations (10), (15), (19) and (32) considering up to 10 different sources (i.e. $N=10$ ). Computation of $\tau_{A C R_{\max }}$ and $\tau_{H}$ has been carried out taking fixed values of $Q(t)$ bounds ( $Q_{H}$ and $Q_{L}$ ), source parameters $\left(A C R_{\max }, A C R_{\min }, P C R, M C R, I C R\right.$ ) and network parameters ( $L C R, N r m, R D F, R I F$ ). Figure 6 shows the analytical results of variation in $\tau_{A C R_{\max }}$ and $\tau_{H}$ as function of $N$ considering example values of $Q_{H}=2000$, $Q_{L}=1000, A C R_{\max }=P C R=L C R=150$ cells/sec., $M C R$ $=0.1$ cells/sec., ICR $=L C R / N, A C R_{\min }=M C R, N r m$ =32, $R D F=1 / 16, R I F=1 / 16, \tau_{b}=\tau_{f}=1$ msec. These selected parameters are also used for determination of $Q_{\max }$ using equations (19) and (32) and $\rho$ is found as a function of 'round trip time' (RTT $=\tau_{f}+\tau_{b}$, ranging from 0-100 msec) with the help of equations (38), (19) and (32). The variation in $Q_{\max }$ as function of $N$ is shown in Figure 7.

Variations of $\rho$ and $P_{\text {loss }}$ are calculated using equations (38) and (40) respectively (utilizing the $Q_{\min }$ and $Q_{\max }$ expressions of equations (32) and (19)) as function of RTT as shown in Figure 8. $\rho$ and $P_{\text {loss }}$ are calculated using equations (38) and (40) respectively as function of $\mathrm{N}$ in the range 1- 10 as shown in Figure 9. Variation of $P_{\text {loss }}$ determined as function of $Q_{\max }$ using equations (40) and (19) is shown in Figure 10.

Referring Figure 6 it can be inferred that the proposed model of the RRM switch offers the benefit of reduced time delay between the instant when $A C R$ attains its maximum value (=ACR $R_{\max }$ ) and the time when $Q(t)$ reaches to $Q_{H}$ with increasing number of sources, however, $\tau_{A C R \max }$ exhibits inverse behaviour. This reduction in $\tau_{H}$ can be useful in faster control of source data rate for reducing cell loss probability due to $Q$ buffer overflow. It may be seen from Figure 7 that $Q_{\max }$ increases rapidly for more than three sources. The link utilization ( $\rho$ ) decreases moderately with increasing RTT and the cell loss probability ( $\left.P_{\text {loss }}\right)$ increases as in Figure 8. The variation of " $\rho$ " and “ $P_{\text {loss }}$ " for different values of " $N$ ” is shown in Figure 9 from which it is clear that higher link utilization is achievable for more number of data sources, however, this is accompanied by affordable increase in $P_{\text {loss }}$ which can of course be minimized by increasing $Q_{\max }$ as shown in Figure 10 . 


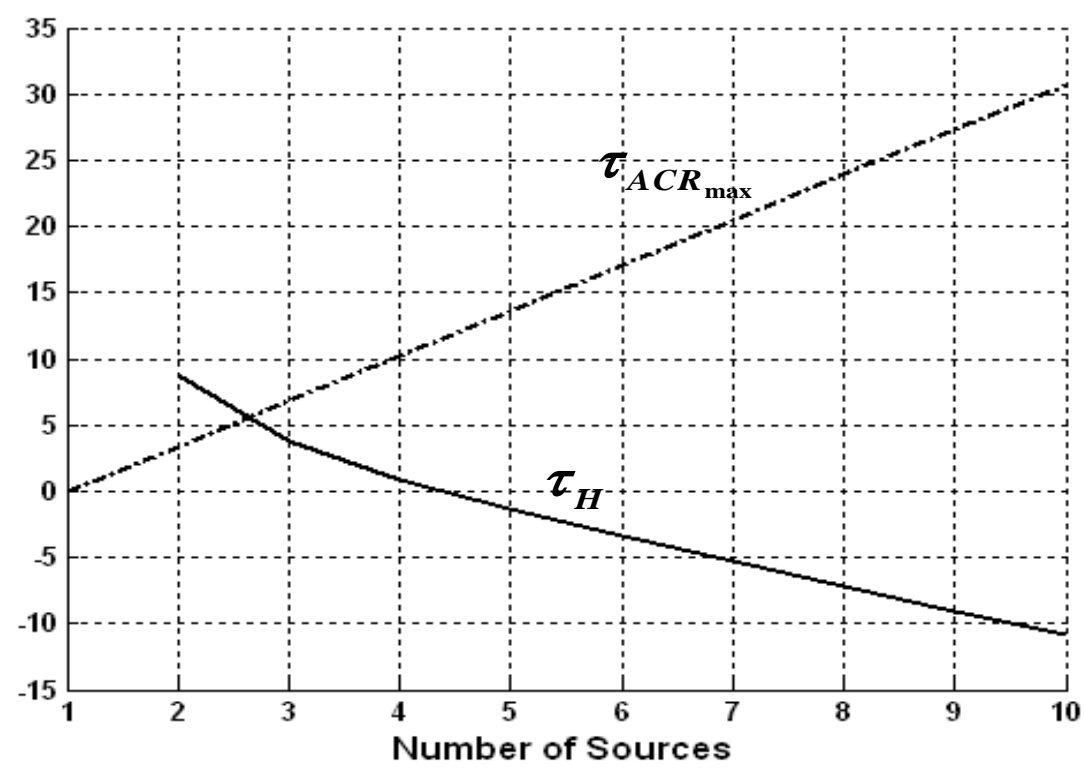

Figure 6. $\tau_{H}, \tau_{A C R \max }$ versus the number of sources, $N$

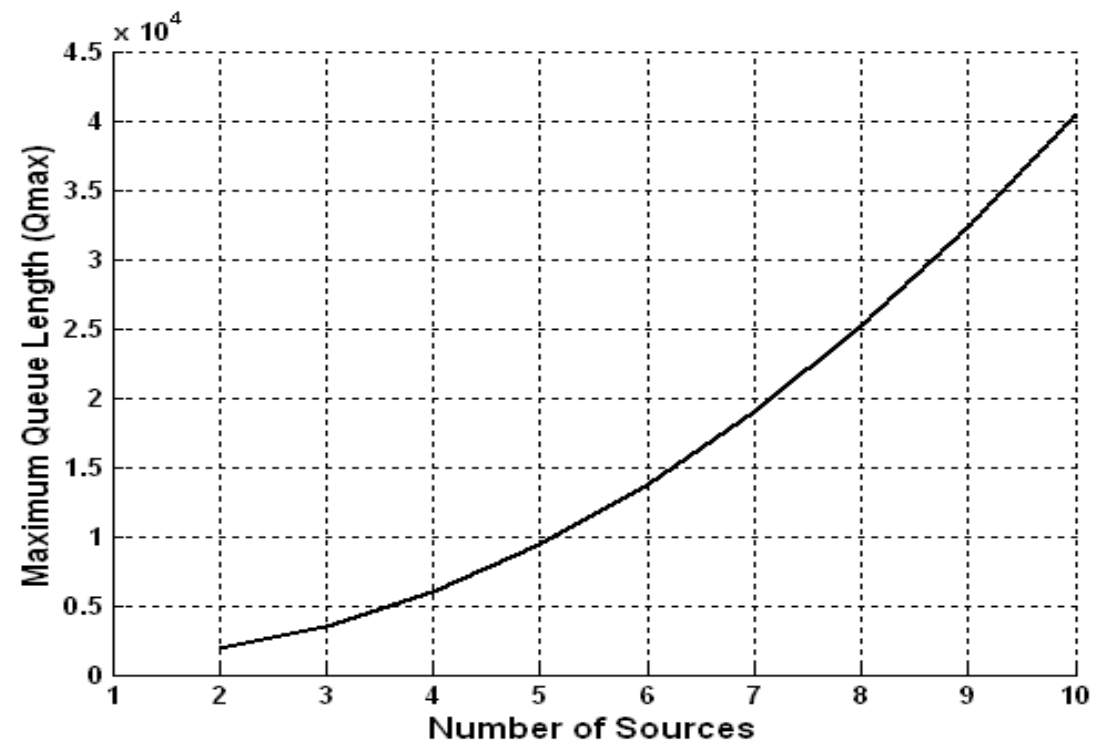

Figure 7. Maximum queue length, $Q_{\max }$ versus the number of sources, $N$

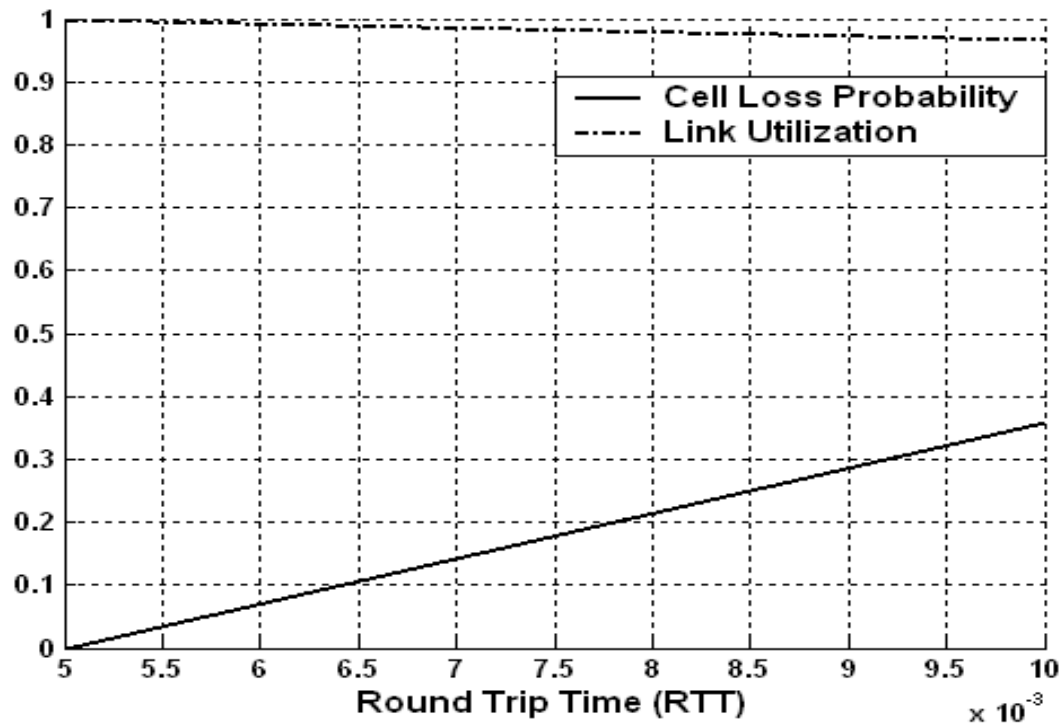

Figure 8. Link Utilization ( $\rho$ ) and Cell Loss Probability $\left(P_{\text {loss }}\right.$ ) versus Round Trip Time (RTT) 


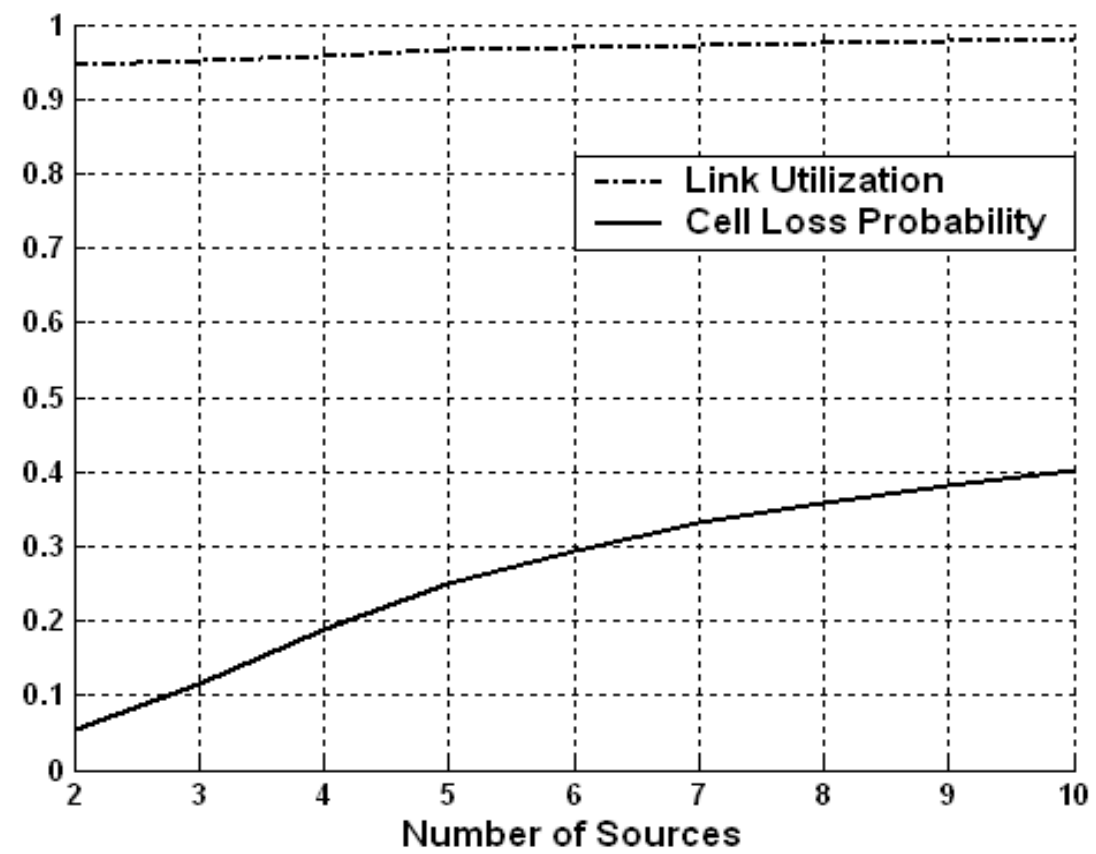

Figure 9. Cell Loss Probability, $P_{\text {loss }}$ and Link Utilization, $\rho$ versus Number of sources, $N$

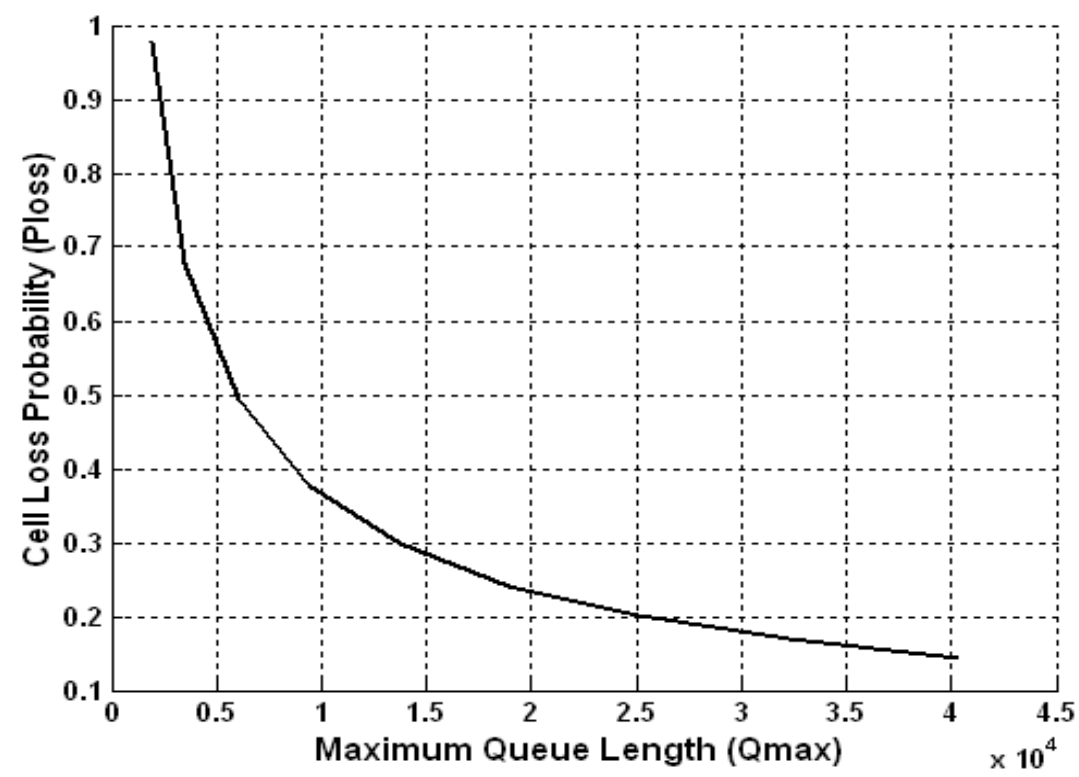

Figure 10. Cell Loss Probability, $P_{\text {loss }}$ versus Maximum Queue Length, $Q_{\max }$

\section{Conclusion}

Modeling of RRM switch has been carried out to facilitate theoretical evaluation of the important parameters of the switch like $Q$ length variation, link utilization factor, cell loss probability as function of round trip time for resource management / number of sources considering linear increment and exponential decay of source data rates. The expressions for different parameters have been developed and results of theoretical computations considering an illustrative example are presented. It is shown that the RRM switch implementation can achieve faster congestion control of the network along with improved link utilization factor which is particularly attractive for large number of sources. Further, the cell loss probability can be reduced by affording higher value of $Q_{\max }$. The presented model can find potential application by using the developed equations for the design of RRM switch specific to a network environment.

\section{References}

[1] Roland Schegg and Brigitte Stangl, Information and Communication Technologies in Tourism 2017, Proceedings of the International Conference in Rome, Italy, January 24-26, 2017.

[2] Mounir Frikha, Ad Hoc Networks: Routing, QoS and Optimization, Wiley-ISTE, 2010.

[3] Charles E. Spurgeon, Joann Zimmerman, Ethernet switches: An introduction to network design with switches, O'Reilly Media, 2013.

[4] Varma and Subir, Internet Congestion Control, Morgan Kaufmann, 2015.

[5] M Mars, A Bianco, R Cigno, and M Munafo, TCP over ABR in ATM networks with variable topology and background traffic, ATM'96 Workshop, Aug, pp. 25-27, 1996. 
[6] Thomas Potsch, Future Mobile Transport Protocols: Adaptive Congestion Control for Unpredictable Cellular Networks, Springer Fachmedien Wiesbaden, 2016.

[7] M Mars, A Bianco, R Cigno, and M Munafo, TCP over ABR in ATM networks with variable topology and background traffic, ATM'96 Workshop, Aug, pp. 25-27, 1996.

[8] D Lapsley and M Rumsewicz, Improved buffer efficiency via no increase flag in EFCI flow control, ATM'96 Workshop, Aug, 1996.

[9] Mischa Schwartz, Broad Band Integrated Networks, Department of Electrical Engineering, Colombia University, New York, N.Y. 1996.
[10] Varadharajan Sridhar and Debashis Saha, Recent Advances in Broadband Integrated Network Operations and Services Management, June, 2011.

[11] $\mathrm{N} \mathrm{T}$ Plotkin and $\mathrm{J} \mathrm{J}$ Sydir, The rate mismatch problem in heterogeneous ABR flow control, INFOCOM'97, Kobe, Japan, pp. 1306-1316, April 1997.

[12] A P Zwart, A fluid queue with a finite buffer and subexponential input, Adv. Appl. Probabil., vol. 32, pp. 221-243, Mar. 2000.

[13] ATM Forum/af-tm-0056.000, PROJECT: ATM Forum Technical Committee Traffic Management Working Group, April 1996. 